\title{
The ninth Japan Bioanalysis Forum symposium
}

Junji Komaba*,1, Jun Hosogi ${ }^{2}$, Harue Igarashi ${ }^{3}$, Hiroshi Kamimori ${ }^{4}$, Keiko Nakai ${ }^{5}$, Takahiro Nakamura ${ }^{6}$, Yosuke Kawai ${ }^{7}$, Yoshihisa Sano ${ }^{8}$ \& Takeru Yamaguchi ${ }^{9}$

${ }^{1}$ Pharmacokinetic Research Laboratories, Ono Pharmaceutical Co., Ltd, Tsukuba, Ibaraki, Japan

${ }^{2}$ Translational Research Unit, R\&D Division, Kyowa Hakko Kirin Co., Ltd, Nagaizumi, Shizuoka, Japan

${ }^{3}$ Bioanalysis, Immunogenicity \& Biomarkers, Pre-Clinical Development Department, GlaxoSmithKline KK, Tokyo, Japan

${ }^{4}$ Research Laboratory for Development, Shionogi \& Co., Ltd, Toyonaka, Osaka, Japan

${ }^{5}$ Drug Metabolism and Analysis Department, LSI Medience Corporation, Kashima, Ibaraki, Japan

${ }^{6}$ Pharmacokinetics and Bioanalysis Center, Shin Nippon Biomedical Laboratories, Ltd, Kainan, Wakayama, Japan

${ }^{7}$ Clinical Pharmacology, Otsuka Pharmaceutical Co., Ltd, Osaka, Japan

${ }^{8}$ DMPK\&Bioanalysis Unit, Sunplanet Co., Ltd, Tsukuba, Ibaraki, Japan

${ }^{9}$ Bioanalysis Group, Osaka Laboratory, Sumika Chemical Analysis Service, Ltd, Osaka, Japan

*Author for correspondence: Tel.: +81 29877 4556; komaba@ono.co.jp

The ninth Japan Bioanalysis Forum symposium took place at tower hall Funabori, Tokyo, Japan, between 6 and 8 February, 2018. Bioanalytical scientists from the pharmaceutical industry, CROs, academia and regulatory bodies had many meaningful and relevant discussions on current topics of interest in bioanalysis. The 3-day symposium featured updated perspectives and experiences on regulated bioanalysis of small and large molecules, biomarker measurement and assessment of immunogenicity, as well as new areas of bioanalytical validation such as quantitative polymerase chain reaction(qPCR) and flow cytometry. There were over 260 participants from six countries, with 23 oral and 11 poster presentations, including the outcomes of Japan Bioanalysis Forum discussion groups. This report summarizes the major discussion topics from the conference.

First draft submitted: 23 July 2018; Accepted for publication: 10 August 2018; Published online: 10 September 2018

Keywords: ADA • bioanalysis • bioanalytical method validation $\bullet$ biomarker $\bullet$ discussion group $\bullet$ JBF

The Japan Bioanalysis Forum (JBF) was established in 2011 as Japan's first and only association with representative experts on bioanalysis from academia, regulatory agencies and industry. The JBF holds an annual open symposium at which various topics and specific challenges related to bioanalysis are discussed. The oral sessions of the ninth JBF symposium were as follows:

- Microsampling in nonclinical toxicity studies;

- Sample preparation automation for bioanalysis;

- Perspectives on ICH M10 bioanalytical method validation guidelines;

- Recent progress on analysis of antidrug antibodies (ADA) in drug development;

- Bioanalysis of therapeutic antibodies by LC-MS and ligand binding assay (LBA);

- Validation for quantitative analysis using flow cytometry, Luminex and qPCR;

- Biomarker measurements and applications for clinical diagnosis.

In addition to the oral sessions, the latest outcomes and recommendations from the JBF Discussion Groups (DGs) were presented in a poster session and discussed by group members and attendees. This report provides a summary of the highlights of the ninth JBF symposium. Presentation materials are available on the JBF website [1].

\section{Microsampling in nonclinical toxicity studies}

First, the final document of ICH S3A Q\&A [2] was presented by Yoshiro Saito (National Institute of Health Science [NIHS]), the topic organizer. Akio Kobayashi (Japan Tobacco) discussed the effects of blood microsampling in a 
rodent toxicity study (current status and future issues) from the perspective of a toxicologist and Yoichiro Nihashi (Shionogi) introduced points to consider in toxicokinetic analysis using microsampling as a bioanalyst. Finally, Jihei Nishimura (Pharmaceuticals and Medical Devices Agency) described the usefulness of microsampling methods and key points in toxicological evaluations from the review authority perspective. Practical use of microsampling methods in toxicokinetic analysis was discussed based on these presentations and explanations of documents and experiences.

\section{Sample preparation automation for bioanalysis}

Tohru Natsume (National Institute of Advanced Industrial Science and Technology) introduced a double-arm humanoid, LabDroid ('Maholo'), that allows various life science experiments to be programmed and performed by a single robotic system using standard laboratory instruments, and without the need for specialized equipment. Dr Natsume proposed that 'Maholo' could be one of the best solutions for migration towards laboratory automation and discussed the concept of 'Robotic Crowd Biology'. Takumi Orikasa (Axcelead Drug Discovery Partners) presented on automation of sample preparation in absorption, distribution, metabolism, and excretion screening, with a description of an automated bioanalytical assay platform with a liquid handling system that provides high efficiency and no concerns of human errors and variations among researchers. Toshikazu Horiuchi (Shin Nippon Biomedical Laboratories [SNBL]) presented a laboratory automation system that can be used under GLP compliance. Bioanalysis workflows have complex and repeated tasks with extensive data handling, which leads to possible human error and the necessity of time for evaluation. Dr Horiuchi addressed the pros and cons of laboratory automation and cautionary points in deployment and operation under regulations.

\section{Perspective on ICH M10 Bioanalytical Method Validation Guidelines}

Akiko Ishii-Watabe and Yoshiro Saito (NIHS) discussed the current status of ICH M10 Bioanalytical Method Validation. The M10 draft technical document has been discussed and revised several times. After the next Kobe meeting in June 2018, the Expert Working Group plans to have internal consultation in each organization. The ICH M10 guidance is then planned to reach step 1 after the US meeting in November 2018. Lindsay King (Pfizer, on behalf of American Association of Pharmaceutical Scientists [AAPS]) gave feedback from discussions at the Weehawken Workshop held in September 2017, sponsored by AAPS, on global collaboration with the European Bioanalysis Forum (EBF) and the JBF. A summary of the output from this workshop was provided for areas in which consensus was not reached. Perspectives were also given on the many challenges that have emerged in the AAPS goal of obtaining harmonized guidelines and harmonized interpretation of these guidelines. Finally, Philip Timmerman (EBF) gave feedback from discussions at the EBF Focus Workshop held in September 2017 on industry input into ICH M10. The industry's top six topics were highlighted: scope, run acceptance criteria, hemolyzed/hyperlipidemic matrix, stability testing, single-duplicate analysis for LBA and critical reagents for LBA.

\section{Recent progress on analysis of ADA in drug development}

Kazuko Nishimura and Akiko Ishii-Watabe (NIHS) gave an overview of points to consider to ensure the reliability of ADA assays, which were discussed in the Japan Agency for Medical Research and Development (AMED) immunogenicity research group [3]. The discussion covered a wide range of topics on ADA assays, including data comparison for cut-off point determination and drug tolerance performed by Japanese pharmaceutical companies and CROs. The outcome of the research group will serve as a basis of the Japanese immunogenicity assessment guidelines in the near future. Sally Saeger (Bristol-Myers Squibb) presented a case study of ADA assay life-cycle management during clinical development. Through the clinical program, ADA assay methodology evolved from first to third generation, with the primary focus to improve drug tolerance. The third generation assay using bead extraction combined with acid dissociation had sufficient drug tolerance. Mitigation of a lack of immunogenicity assessment by the clinical team was provided by reanalysis of banked in-study samples in Phase I, using a fitfor-purpose validated assay. Maiko Adachi (Kyowa Hakko Kirin) introduced a recent challenge to improve drug tolerance in both clinical and nonclinical stages. The pros and cons of the plate-/bead-solid phase extraction with acid dissociation and precipitation and acid dissociation methods [4] were presented in a comprehensive manner. Brief results from a novel immune complex ADA assay for nonclinical studies were also presented. 


\section{Bioanalysis of therapeutic antibodies by LC-MS \& LBA}

First, Steve Lowes (Q2 Solutions) explained the concept of the AAPS White Paper issued in 2015 [5]. Dr Lowes presented current discussions and new technologies (e.g., hybrid LC-MS and high-resolution MS) for bioanalysis of therapeutic antibodies by LC-MS, and concluded that LC-MS is now well established as a complementary tool for LBA approaches. Roland Staack (Roche, on behalf of EBF) presented feedback from the EBF topic team dedicated to 'Free/total drug quantification' and from a workshop held at the annual EBF symposium in Barcelona 2017. Both free (active) drug and total drug data are important for understanding and interpretation of PK/PD data and drug clearance. Third, Nozomu Kato (Mitsubishi Tanabe Pharma, on behalf of the JBF Task Force: Large Molecule LC-MS) introduced commentaries and proposals on the LC-MS method for therapeutic antibodies, based on discussions of the JBF Task Force. Dr Kato recommended referral to the LC guideline [6] for preparation procedures and evaluation items, since the platform is LC-MS. On the other hand, for acceptance criteria (accuracy and precision $[A \& P])$, he suggested referral to the LBA guideline [7], since therapeutic antibodies are generally analyzed using the LBA method. Finally, Noriyuki Danno (CMIC Pharma Science) introduced analytical methods (LC-MS and LBA) for therapeutic antibodies and discussed their measurement from the viewpoint of detection sensitivity and operating efficiency, with the conclusion that the analytical method should be selected depending on the purpose.

\section{Validation for quantitative analysis using flow cytometry, Luminex \& qPCR}

Yoshitaka Hirasawa (Ina Research) introduced the principles of analysis in flow cytometry and Luminex technology for endogenous substance analysis, and also discussed design of validation studies appropriate for simultaneous detection of multiple endogenous substances, including other points that should be taken into account. Dr Hirasawa made a point of showing that a fit-for-purpose approach is appropriate for analysis of multiple endogenous substances using these platforms. Asako Uchiyama (SNBL) presented design considerations for validation of qPCR for bioanalysis (see details in the DG paragraph).

\section{Biomarker measurements \& application for clinical diagnosis}

Yoshiro Saito (NIHS) briefly introduced the current situation of biomarker measurement from the regulatory viewpoint $[8,9]$ and presented part of a questionnaire survey on current practice of biomarker measurement collected from 37 pharmaceutical companies in Japan. Dr Saito also mentioned the further plan, including his own ideas, for preparation of a White Paper based on this survey. Kenji Nakamaru (Daiichi Sankyo) discussed the many challenges in development of biomarker assays, including patient selection and companion diagnostic (CDx) assays in the early and late stages of drug development. Dr Nakamaru mentioned that selecting and building a close relationship with the right partner with the expertise to transition the assay into an investigational use only kit and planning every stage of development, including regulatory and marketing in alignment with drug development, are keys to success. Hirohisa Matsushita (Nichirei Biosciences) described current issues in development of CDx from the perspective of an in vitro diagnostics manufacturer, and noted that the most challenging issue is that samples used for cut-off evaluation are insufficient and limited by informed consent in clinical studies. Therefore, alignment of development timing with the drug is important for CDx development. Akira Nakayama (Ajinomoto Co., Ltd) discussed the kinds of data that should be acquired from the regulated bioanalysis view through development of AminoIndex ${ }^{\circledR}$ Cancer Risk Screening $\left(\right.$ AICS $^{\circledR}$ ), which measures amino acids as biomarkers of cancer risk. Amino acids levels in plasma do not change markedly, and so it was suggested that quality control of analytical methods for these markers should be carried out at a level different from the $\pm 15 \%$ in bioanalytical method validation guidelines. Dr Nakayama introduced trial and error in these measurements, including notes on analytical method development and management of specimens and standard solutions.

\section{JBF DGs}

DG promotion committee (chair: Yoshihisa Sano; Sunplanet) organized this session. The members of seven DGs (DG 28-34) introduced their activities and exchanged views on a broad range of bioanalytical issues with attendees in poster sessions on the evening of day 2 and morning of day 3.

DG28 worked on 'Revisiting A\&P criteria'. The group consisted of nine members (leader: Makoto Niwa; Nippon Kayaku). The goal was to draw attention to fit-for-purpose A\&P criteria and to discuss the principles in setting criteria in early phase metabolite quantification for assessing the probability of metabolite safety testing. 
Recommended metabolite-testing workflows and statistically justified metabolite-risk assessments were shared with the attendees.

DG29 focused on 'Microsampling: proposals for planning and implementation'. The group consisted of seven members (leader: Yoichiro Nihashi, Shionogi). To provide practical information to Japanese companies who wish to apply microsampling in regulated bioanalysis, the group discussed the following issues in conducting bioanalysis by LC-MS: 'Sample dilution with appropriate solvents', 'Sample storage', 'Partial validation in transferring to microsampling', 'Suitable ratio of anticoagulant in blood sampling' and 'A\&P of pipetting in liquid handling'.

DG30 continued discussion of 'Applications of imaging mass spectrometry in drug discovery research', which was started in 2016. The group consisted of five members (leader: Yukari Tanaka; Shionogi). The many exciting topics included technological issues of how to create a calibration curve for quantification, techniques for increasing sensitivity, normalization methods for tissue images and the capability of desorption electrospray ionization imaging. Applications of imaging mass spectrometry in pharmacokinetic evaluation, PK/PD analysis and pharmacological and safety biomarker detection were also discussed.

DG31, which consisted of seven members (leader: Koji Arai; LSI Medience), presented outcomes of the topic 'Quantitative analysis of endogenous large molecules by LC-MS', following the work of DG08 (2014), DG15 (2015) and DG25 (2016) over 3 years. Topics focusing on protein quantification by LC-MS were discussed; for example, confirming the amino acid sequence of a target protein and digested peptides, selection of appropriate peptides for quantitation and methods of analysis.

DG32 discussed 'Automated sample preparation in regulated LC-MS bioanalysis'. The group consisted of eight members (leader: Takumi Noda; Ono Pharmaceutical). Introduction of automation was recognized as one of the great opportunities to improve the efficiency of bioanalysis. There was a discussion of the circumstances of automation that has been utilized or is anticipated, and much time was spent on discussing obstacles for introduction of automation and how to overcome them. The current status of utilization of automation in regulated bioanalysis in Japanese companies was also discussed.

DG33 summarized the points to consider when performing qPCR for bioanalyses. The group consisted of seven members (leader: Asako Uchiyama, SNBL). The method validation protocol of qPCR for nucleic acids has been under discussion due to the variability in this protocol, including nucleic acid extraction, sample preparation and algorithms used by qPCR machines. The basics of qPCR and significant protocol variabilities that contribute to differences in validation and acceptance criteria were presented. Taken together, the group suggested that the validation protocol should be planned study by study, rather than establishing common acceptance criteria. The list of items to be validated requires further discussion.

DG34 discussed the topic of 'Ligand binding assays using commercial immunoassay kits', following the work of DG26 (2016). The group consisted of nine members (leader: Hiroyuki Shimizu; Mitsubishi Tanabe Pharma). Some commercial kits are not ready-to-use for regulated bioanalysis, and users may need to optimize them for a specific purpose. The group mainly focused on method development, method validation, analysis of study samples and multiplex assays for commercial kits, and presented an overview of their discussion.

\section{Conclusion}

Bioanalysis plays a significant role in drug discovery and development. The ninth JBF symposium provided a great opportunity for attendees to share and exchange scientific knowledge of bioanalysis. The symposium featured the latest topics in regulated bioanalysis, biomarker measurements, ADA assays and new technologies. As in the past, the ninth JBF conference had highly interactive and diversified topics, and attracted attendees from overseas and from Japan. The JBF is the sole organization involved in regulated bioanalysis in Japan, and has been expanding its scope to cover all bioanalysis-related issues. The JBF tenth anniversary symposium will be held in Yokohama, Japan between 12 and 14 February, 2019.

Financial \& competing interests disclosure

The authors have no relevant affiliations or financial involvement with any organization or entity with a financial interest in or financial conflict with the subject matter or materials discussed in the manuscript. This includes employment, consultancies, honoraria, stock ownership or options, expert testimony, grants or patents received or pending or royalties.

No writing assistance was utilized in the production of this manuscript. 


\section{References}

1. Japan Bioanalysis Forum. http://bioanalysisforum.jp/en

2. ICH. Harmonized Guideline: ICH S3A Q $\circlearrowleft$ A. Questions and Answers: Note for Guidance on Toxicokinetics: the Assessment of Systemic Exposure - Focus on Microsampling. http://www.ich.org/fileadmin/Public_Web_Site/ICH_Products/Guidelines/Safety/S3A/S3AIWG_Step4_2017_1116.pdf

3. Ishii-Watabe A, Shibata $\mathrm{H}$, Nishimura $\mathrm{K}$ et al. Immunogenicity of therapeutic protein products: current considerations for anti-drug antibody assay in Japan. Bioanalysis 10(2), 95-105 (2018).

4. Zoghbi J, Xu Y, Grabert R et al. A breakthrough novel method to resolve the drug and target interference problem in immunogenicity assays. J. Immunol. Methods 426, 62-69 (2015).

5. Jenkins R, Duggan JX, Aubry AF et al. Recommendations for validation of LC-MS/MS bioanalytical methods for protein biotherapeutics. AAPS J. 17(1), 1-16 (2015).

6. Japanese Ministry of Health, Labour and Welfare. Guideline on Bioanalytical Method Validation in Pharmaceutical Development (2013).

7. Japanese Ministry of Health, Labour and Welfare. Guideline on Bioanalytical Method (Ligand Binding Assay) Validation in Pharmaceutical Development (2014).

8. Arnold ME, Booth B, King L, Ray C. Workshop report: crystal city VI - bioanalytical method validation for biomarkers. AAPS J. 18 (6), 1366-1372 (2016).

9. Biomarker Assay Collaborative Evidentiary Considerations Writing Group, Critical Path Institute (C-Path). Points to Consider Document: Scientific and Regulatory Considerations for the Analytical Validation of Assays used in the Qualification of Biomarkers in Biological Matrices. https://healthpolicy.duke.edu/sites/default/files/atoms/files/white_paper_6_14_17_0.pdf 
